\section{HOW DEEP IS YOUR LOVE: AWARENESS MONITORING DURING GENERAL ANESTHESIA}

\author{
Radmilo Janković ${ }^{1,2}$, Danica Marković ${ }^{1}$, Biljana Stošić $c^{1,2}$ \\ ${ }^{1}$ Center for Anesthesiology and Reanimatology, ClinicalCenter of \\ Niš, Serbia \\ ${ }^{2}$ Department for Anesthesiology and Intensive Care,School of Me- \\ dicine, University of Niš, Serbia
}

\section{HOW DEEP IS YOUR LOVE: MONITORING BUDNOSTI TOKOM ANESTEZIJE}

\author{
Radmilo Jankovićl, ${ }^{1,2}$ Danica Markovićl, Biljana Stošić1, \\ ${ }^{1}$ Centar za anesteziologiju i reanimatologiju, Klinički centar u Nišu, \\ Niš, Srbija \\ ${ }^{2}$ Katedra za anesteziologiju i intenzivnu negu, Medicinski fakultet, \\ Univerzitet u Nišu, Niš, Srbija
}

\section{Abstract}

The 5th National Audit Project (NAP5) has established that the incidence of accidental awareness during general anaesthesia is about 1 in 15414 general anesthesia. In total, about two-thirds of accidental awareness during general anaesthesia happened during the so-called "dynamic“ phase of anesthesia. The stages of consciousness are often very short and that implies that the used monitoring must be able to detect even a short phase of consciousness. Administration of neuromuscular blockators makes monitoring of consciousness difficult, therefore currently the most reliable methods of monitoring are: IFT, BIS monitoring and maintenance of ETAG > 0.7 MAC. Certainly, none of these methods can be used independently from the experienced anesthesiologists and clinical methods for assessing accidental awareness during general anaesthesia.

Key words: awareness; monitoring; general; anesthesia

\section{Introduction}

A ccording to some studies, the incidence of accidental awareness during general anesthesia (AAGA) showed an extremely high frequency, even 1-2 per 1,000 general anesthesia, while some other studies had suggested a much lower incidence of AAGA, about 1 in 14500 of general anesthesia $^{1-4}$. Pandit et al. under the 5th National Audit Project (NAP5) found in 2011. that the number of AAGA is about 1 in 15414 of general anesthesia. Two thirds of AAGA occurred during the so-called „dynamic“ phase of anesthesia, especially during or immediately after induction of anesthesia. The stages of consciousness during anesthesia were extre-

Autor za korespondenciju: Radmilo Janković, Klinički centar u Nišu, Bulevar dr Zorana Đinđića 48, 18000 Niš, Srbija, Telefon: 065/3349729, E-mail: jankovic.radmilo@gmail.com

\section{Sažetak}

Istraživanja u okviru The 5th National Audit Project (NAP5) su utvrdila da se prisustvo slučajne svesti tokom opšte anestezije dešava jednom u 15414 slučajeva. Ukupno oko dve trećine slučajnih svesti tokom opšte anestezije se dešavaju tokom takozvanih dinamičnih faza anestezije. Prisustvo svesti obično jako kratko traje, što ukazuje na to da monitoring mora biti osposobljen da detektuje čak $i$ kratke faze svesti. Administracija neuromišićnih blokatora otežava monitoring svesti, te se najpouzdanijim monitoringom smatraju: izolovana tehnika nadlaktice, BIS monitoring $i$ održavanje ETAG iznad 0,7 MAC. Svakako ni jedna od ovih metoda se ne sme koristiti izolovano od iskustva anesteziologa i kliničkih metoda za procenu stanja svesti tokom opšte anestezije.

Ključne reči: budnost; monitoring; opšta anestezija

mely short, therefore the used monitoring must be able to detect short phases of consciousness 5 .

Presence of awareness is classified by authors Griffith and Jones as follows: presence of awareness with complaints of pain observations, the presence of consciousness with a clear reply but absence of pain, the presence of consciousness or "alertness" (the ability to respond to simple verbal commands), the absence of a clear answer and pain but possible presence of memory, lack of a clear response but with the presence of memory about intraoperative events and lack of awareness ${ }^{6,7}$.

Three main risk factors are described: trauma, cesarean section, and cardiovascular surgery. In the case of these surgeries anesthesia is intention-

Corresponding author: Radmilo Janković, Clinical center in Niš, Bulevar dr Zorana Đinđića 48, 18000 Niš, Serbia, Telephone: 065/3349729, E-mail: jankovic.radmilo@gmail.com 
ally maintained shallow in order to avoid serious cardiovascular depression or birth defects. About 95\% of AAGA cases are associated with human mistake or failure of anesthesiological device or failure of monitoring devices ${ }^{8}$.

Ideal anesthesia is achieved by using drugs such as hypnotics, analgesics, neuromuscular relaxants and implies the absence of consciousness, antinociception and immobility. Studies have clearly demonstrated that some patients require significantly higher doses of medications in order to achieve and maintain an adequate level of anesthesia ${ }^{8}$.

Intraoperative awareness with clear motor response is associated with post-traumatic stress disorder, whose incidence of about $0.15 \%{ }^{9}$. In order to reduce the incidence of awareness with the patients response, there are discussions in scientific circles which monitoring should be used during inhalational anesthesia. Recommendations regarding appropriate monitoring are more and more implemented in national clinical practice guidelines in order to improve anesthesia, reduce the amount of anesthetic that is used in a patient, reduce costs, improve postoperative recovery and reduce the incidence of awareness ${ }^{10}$.

The level of awareness is mostly determined by the degree in which a person responds or not to the growing stimulus. Failure of verbal or motor response after the administration of hypnotics or narcotics (without administration of muscle relaxant) even after painful surgical stimulus is considered to be a patient in a sufficiently deep anesthe$\operatorname{sia}^{11}$. The fact is that the administration of neuromuscular blockers prevents the motor response of patients and therefore makes experienced anesthesiologists assessment difficult of whether the patient is conscious or not. A large number of researchers believe that even anesthesia guided with appropriate monitoring does not provide complete certainty that the patient will lose consciousness during surgery ${ }^{10}$. In such cases, the determination of the consciousness level is based on the anesthesiologists experience and on the use of anesthesia depth monitoring such as isolated forearm technique (IFT) or processed EEG monitor (pEEG) $)^{11,12}$.

\section{Clinical techniques and standard monitoring of aaga}

Standard clinical methods for AAGA assessing are: response to the given commands, gestures, eye- lash reflex, perspiration, pupil response or change of its diameter and watery eyes ${ }^{7,8}$. The most commonly used scoring represents PRST or Evans's index, which includes $\mathrm{P}$ (systolic blood pressure), $\mathrm{R}$ (heart rate), $\mathrm{S}$ (sweating) and $\mathrm{T}$ (tears) ${ }^{7,13}$. Index score ranges from 0 to 8 , however, it rarely exceeds the mean value, which indicates the deficinency of the scoring system in daily practice. It is believed that monitoring of heart rate and blood pressure with assessment of pupil size is the most useful for the assessment of adequate analgesia and depth of anesthesia. High frequency fluctuations in heart rate i.e. changes in heart rate with the frequency of ventilation (respiratory sinus arrhythmia, RSA) reflect the level anesthesia depth. Such monitoring is useful but depends on the extent to which the autonomic nervous system of the patient is preserved as well as on the health of the patients cardiovascular system. Beta blockers, conduction abnormalities, autonomic neuropathy and sepsis may influence this type of monitoring ${ }^{7,8,14}$.

Standard monitoring includes electrocardiogram, blood pressure, heart rate, capnography and end tidal anesthetic analyzer. There are no major clinical trials which determine connection between standard clinical techniques and standard monitoring with the incidence of AAGA occurrence ${ }^{8}$.

\section{Most frequently used aaga monitoring}

In order to minimize intraoperative awareness of the patient, anesthetist has three available options: IFT, BIS monitoring and maintenance of ETAG $>0.7 \mathrm{MAC}^{12}$.

End-tidal anesthetic gas monitoring (ETAG)

Anesthetic agents are titrated by their minimum alveolar concentration (MAC) and the hemodynamic response. MAC is defined as the anesthetic concentration at 1 atmosphere which causes immobility in $50 \%$ of patients exposed to surgical stimulation. However, it turned out that MAC has a poor correlation with the degree of hypnosis ${ }^{12}$.

ETAG monitoring alarms represent a reliable way of securing sufficient quantities of volatile anesthetics for a specific patient. Audible signals are heard if the minimum alveolar concentration reaches $<0.7$ or $>1.3^{1}$. Several studies have concluded that BIS monitoring is not better than ETAG monitoring in the prevention of patient awareness during anesthesia ${ }^{1,9,10}$. 


\section{Isolated forearm technique (IFT)}

In a performance of the isolated forearm technique (IFT) monitoring Tourniquet is placed on one forearm and inflates above arterial pressure before neuromuscular relaxant is injected into one of the peripheral veins. For this reason neuromuscular blocking agent does not reach neuromuscular junctions located below the Tourniquet and therefore reflex responses or responses to commands issued by the anesthesiologist can be observed ${ }^{10,15}$.

IFT is considered in today's practice to be the gold standard for awareness monitoring in the presence of neuromuscular blockade ${ }^{16}$. It is the fact that the use of IFT showed that inadequate levels of anesthesia has not been accompanied by heart rate change, sweating, and the production of tears in practice ${ }^{17}$.

There are a number of reasons why IFT monitoring cant be effective in everyday practice. For example, anesthetic can occasionally cause catatonic „locked“ status (catatonic locked-in state) in the anesthetized but partially conscious patients ${ }^{12,18}$. Also, there may be a number of technical problems such as cuff failure, unexpectedly long clearance of muscle relaxants, limb ischemia, etc. ${ }^{18}$. The negative side of this technique is also the so-called state of dysanesthesia, in which the patient's perception is partly separated from the feeling, so the patient responds to commands but does not move his hand spontaneously during surgery ${ }^{19}$. Some clinicians believe that the motor response during the operation is the late sign of awareness and that IFT has no importance in the prevention of consciousness ${ }^{8}$.

Russel has explained in his editorial some of the misconceptions associated with this method after many years of experience with the use of IFT. According to experience, it is possible to keep the inflated Turniquet up to 40 minutes without the appearance of ischemic nerve block, even an easy monitoring of neuromuscular integrity through a peripheral nerve stimulator is recommended. He also believes that the ischemic nerve block recovers a few minutes after the release of Tourniquet. Use of almost all muscle relaxants (curare, alcuronium, atracurium, vecuronium, lower doses of pancuronium, $0.05 \mathrm{mg} / \mathrm{kg}$ ) was not accompanied with paralysis of the limbs after Tourniquet release ${ }^{15}$.

Not too many anesthesiologist use the IFT technique since they believe that this technique is difficult, and that this is a technique that takes too much time and distracts attention from other monitoring ${ }^{19}$. It is evident that IFT method as the monitoring technique is used by only 14 of over 8,000 clinicians in England ${ }^{20}$.

Reported cases of wakefulness during the use of IFT techniques are rare, except for a few reported cases of discomfort. Studies which used an IFT method showed that many patients may be aware and able to respond to commands and not to have post-operative memories ${ }^{10,21}$.

Of course, extensive studies are needed in order to determine the value of IFT monitoring in everyday practice.

\section{Monitoring of brain electrical activity}

Devices designed to monitor electrical activity of the brain are mainly based on information obtained from the electrodes on the forehead of the patient. After amplification and conversion of EEG signals an index is obtained and it generally ranges from 0 to 100. This index reflects the state of mind, ie the state of vigilance, sedation, light anesthesia and deep anesthesia ${ }^{7,8}$

One of the oldest and most basic monitoring of this type is the monitoring of spontaneous EEG activity with 19 electrodes. However, besides it requiring a longer time of placement and taking longer to start monitoring, it is evident that different anesthetics give a different EEG picture and therefore the interpretation is difficult. Also, various events (hypotension, hypercarbia, etc.) have the consequence of EEG changes ${ }^{22}$. Similar to this monitoring is the compressed spectral analysis (CSA). However, in addition to other numerous deficiencies there is also the fact that it cant be used independently ie. without the active monitoring of clinical parameters ${ }^{7,8,23}$. Some other possible solutions for monitoring the electrical activity of the brain are: EEG with compressed spectral analysis, cerebral function monitor (CFM), cerebral function analysis monitor (CFAM), entropy (which processes EEG signals and FEMG signals with the help of the Entropy algorithm), Cerebral state monitor / Cerebral state index (CSI), etc. ${ }^{7,8,23,24}$

\section{Processed EEG monitoring}

Propofol, thiopentone and inhaled anesthetics produce similar EEG changes as a result of en- 
hancement of their concentration in the brain tissue and deepening of the sedation and anesthesia depth. With the increase of anesthesia depth the following changes in the EEG signal happen: initial increase of high-frequency components, then increase of the low frequency components, increase of the amplitude of EEG waves, increase of the regularity of the EEG signal, burst suppression in deep anesthesia and isoelectric „straight line“ in very deep anesthesia.

The Bispectral Index (BIS) monitor (Covidien, Boulder, CO) keeps track of frontal EEG channel with the aim to calculate the number between 100 (alertness) and 0 (not detectable brain activity) and therefore measure the patient's level of consciousness ${ }^{9}$. BIS index represents an algorithm which converts the single-channel EEG into the index of the hypnosis degree. There are several intraoperative events that are not related to the amount of anesthetics and which cause a rapid change in BIS values, such as: brain ischemia, air embolus, unrecognized hemorrhage, etc. ${ }^{7,8}$. The values of BIS index 60-80 indicate that the patient can more easily respond to mechanical stimulation, while the value of $45-60$ is associated with a low probability of a clear answer. BIS level below 45 is associated with the state of deep hypnosis. Russel et al and Zand et al have showed that the BIS value below 60 does not mean a little chance of vigilance without an answer when compared to the IFT results $s^{9,10,15,16}$.

Cochrane review declared that BIS-guided anesthesia can reduce the risk of intraoperative awareness in surgical patients who are at high risk of awareness ${ }^{25}$. NICE has published the Diagnostics Guidance report (2012) which recommends that the pEEG monitoring is used in all the patients who were under the influence of total intravenous anesezije (TIVA) ${ }^{26}$.

Recommendation when using BIS monitoring in the case of inhalation anesthesia and the use of opioids is to maintain an inhalational anesthetic flow rate so that the BIS monitor shows 45-60. However, the research by Russel et al has pointed to the fact that 11 out of 34 patients responded to the command during the operation when monitored by IFT in spite of the fact that BIS has been maintained in the range 55-60. BIS has also demonstrated the level of awareness 660 times even though IFT showed no motor response to the command. The largest number of motor responses has been observed when the BIS ranged 46-50 and $56-60^{10}$.

Meta-analyzes have pointed to the fact that the BIS monitor can greatly reduce the use of anesthetics, reduce the time to extubation, lead to faster recovery from anesthesia as well as reduce the incidence of nausea and vomiting and reduce the level of intraoperative awareness ${ }^{9,25,27}$. However, Mashour et al have showed in their study that the use of the BIS monitor is not associated with a reduction in recovery time, as well as with a reduced incidence of nausea and vomiting 9 .

Zand et al have pointed out that BIS is not a reliable monitor of depth of anesthesia when it comes to caesarean section and sevoflurane anesthesia. It takes less than recommended levels of BIS to avoid IFT responses during laryngoscopy, intubation and skin incision ${ }^{16}$.

A long period of deep hypnosis (BIS < 40) can increase postoperative morbidity and mortality. Also, extended deep hypnosis may have a shortterm negative impact on recovery from anesthesia, as well as cause postoperative delirium and cognitive disorders. It can disrupt the immune system and accelerate the emergence of infections and tumor growth. It is therefore of great importance to know the BIS values in certain MAC concentrations of inhaled anesthetics. Kim et al. have compared BIS profiles of the study participants with the equipotent doses of isoflurane, sevoflurane and desflurane (1MAC) during the maintenance phase of anesthesia. The duration of deep hypnosis (BIS $<40$ ) was significantly shorter, while the duration of adequate hypnosis (BIS 40-60) was significantly longer in the use of sevoflurane in relation to desflurane. Desflurane thus causes a greater depth of hypnosis in relation to the dose of sevoflurane, which in theory, should cause the same level of anesthesia ${ }^{12,28}$. Ketamine and sometimes $\mathrm{N}_{2} \mathrm{O}$ cause EEG activation, thus complicate an adequate interpretation of BIS.

In the case of epidural anesthesia, clinical trials have shown that sedation of such patients requires much smaller MAC of Sevoflurane $(0.59 \%$ compared to $0.92 \%$ ) to achieve an adequate $\mathrm{BIS}^{29}$. Panchal et al. have shown on the pediatric population that BIS values range $71.65 \pm 13.5$ and the end-tidal concentration at $6.41 \pm 0.67$ at induction of anesthesia with sevoflurane, kept at 40 (end-tidal concentration $7.08 \pm 0.69$ ) during intubation, 
ranged between 40 and $60(1.5 \pm 0.64)$ during maintenance of anesthesia until extubation was done at the BIS value of $75(0.14 \pm 0.27)^{30}$.

It is important to note that among patients with dementia value of BIS index (alertness) is lower than in patients who do not have such pathology ${ }^{31}$. Although BIS represents a useful monitoring for the assessment of anesthesia depth, further studies are necessary in patients with congenital disorders of the central nervous system since BIS can give abnormal responses which do not correspond to the specific stage of anesthesia. This is particularly important in the case of the administration of high doses of sevoflurane which can result in epileptogenic activities ${ }^{32}$.

\section{Other metods of aaga monitoring}

In addition to the most commonly used monitoring methods of patients awareness in practice, there are numerous other methods which are less frequently used. One method is to measure the conductivity of the skin, ie. the level of sweat production. It is believed that the conductivity of the skin is initially low and increases with the depth of anesthesia, while it decreases again during the skin incision. One of the main disadvantages of this kind of monitoring is the fact that some conditions and medications (eg, atropine) may interfere with this kind of monitoring 8 .

Spontaneous surface electromyogram (SEMG) monitors the different muscle groups, especially facial, abdominal and neck muscles in patients who are not fully paralyzed. It is considered that the branches of these nerves are incompletely affected by the neuromuscular blockade. The most commonly used electrode is placed above the frontal muscle and this record is called frontalis electromyogram (FEMG). It is considered that FEMG falls during the sufficient depth of anesthesia ${ }^{7,8}$. It is also possible to measure the lower oesophageal contractility (LOC) since the smooth muscles in the lower part of the esophagus retain activity even after complete muscular paralysis. Two types of measurements are lower oeasophageal spontaneous contractility (SLOC) as well as provoked lower oeasophageal contractility (PLOC) ${ }^{8}$. SLOC are induced by emotions and stress in conscious person, while PLOC is the result of the sudden esophagus distension after the arrival of the food bolus. In hospital settings, PLOC is caused by inflating a balloon catheter in the lower part of the esophagus.

Narcotrend (Monitor Technik, Bad Bramstedt, Germany) represents an EEG monitor which is designed to measure the depth of anesthesia in the form of an index that ranges from 100 (awake) to 0 (electrical silence). It provides the following information: current Narcotrend status and index, trend (cerebrogram), raw EEG signal and several EEG derived parameters. Information are obtained through two electrodes that are placed on the forehead of the patient and third, reference electrode. After the elimination of the artifacts, informations are classified as follows: A (awake), B (sedated), C (light anesthesia), D (general anesthesia), E (general anesthesia with deep hypnosis), F (general anesthesia with increasing burst supression). Narcotrend stages $\mathrm{D}$ and $\mathrm{E}$ are equivalent to BIS values of 64 to $40^{8,33}$. Newer devices allow transfer of the alfabet scale into a number scale similar to the BIS index ${ }^{7,8}$. Conducted studies have indicated that Narcotrend did not effectively distinguish awareness and unconsciousness especially in the case of neuromuscular relaxants use, however it is believed that more extensive studies must be carried out in order to preserve the adequate evaluation ${ }^{34,35}$.

The Patient State Analyzer Index (PSI; Physiomnetrix, North Billerica, MA) is obtained by processing the 4-channel EEG and rejecting the artifacts. PSI has a range of 0 to 100 , similar to BIS and Narcotrend monitors. It represents a clinically valid measure of measuring the effects of anesthesia and sedation and is designed specifically for the needs of operative and intensive care. It is known that PSI adequately demonstrates a lack of awareness but there are no studies which show that PSI is really an adequate means of measuring intraoperative awareness ${ }^{7,8,36}$.

SNAPII (Everest Biomedical Instruments, Chesterfield, MO) calculates the index from the single-channel device that records the patient's EEG and subsequently processed it through its unique SNAP algorithm. SNAP index is also in the range between 100 and 0 . Rare studies have indicated that SNAP is perhaps even more sensitive than BIS in detecting AAGA, especially after general anesthesia with sevoflurane and $\mathrm{N}_{2} \mathrm{O}$ on 1 to $1.5 \mathrm{MAC}^{8,37}$.

Another monitoring of the depth of anesthesia is Evoked brain electrical activity monitors, which 
measure electrical activity in certain areas of the brain in response to stimulation of specific sensory nerve pathways. Examples of such monitorings are:

Somatosensory evoked potentials (SSEP) - supramaximal stimulus is applied to peripheral nerve while electrodes are placed over the corresponding sensory area. Many anesthetics increase latency and reduce the amplitude on dose dependent manner. Etomidate increases the amplitude.

Visual evoked potentials (VEP) - diodes are built in the special glasses, and in that way stimulation of the optic nerve is done. EEG electrodes record potentials in the area of the occipital bone. Many anesthetic increase latency and reduce amplitude on the dose-dependent manner.

Auditory evoked potential (AEP) - represents the transfer of electrical activity from cochlea to cortex ${ }^{8}$.

\section{The most effective methods of moitoring aaga}

There are a large number of studies which investigate the effectiveness of different types of monitoring in the presence of the certain level of intraoperative awareness. Mashour et al have showed by post hoc analysis that patients' awareness was present in $0.12 \%$ in a group where the level of consciousness was monited by ETAG method, $0.05 \%$ in the group that was monitored by BIS and $0.15 \%$ in the group where there was no monitoring of patients intraoperative awareness ${ }^{9}$. Avidan et al have showed clear intraoperative awareness in $0.24 \%$ of patients in the BIS group compared to $0.07 \%$ of patients in the ETAG group. Also it was noted that there was $0.66 \%$ of possible intraoperative awareness in patients in the BIS group as well as $0.28 \%$ in the ETAG group. In both cases the superiority of the BIS protocol has not been proven. There was no difference in the incidence of postoperative complications between the two groups ${ }^{1}$.

Anderson et al. have investigated the effectiveness of depth of anesthesia monitoring during the wash in and wash out phase by sevoflurane by BIS monitoring, auditory evoked potential index (AAITM) and ETAG sevoflurane. Ten patients without premedication have received $0.05 \mathrm{mg}$ of fentanyl and $30 \mathrm{mg}$ of propofol as coinduction. After a three-minute preoxygenation loop system has been primed with $6 \mathrm{~L} / \mathrm{min} 8 \%$ sevoflurane. The patient is indicated to deeply breathe two to three times and then to resume normal breathing through the mask. During anesthesia gas flow was increased to $7 \mathrm{~L} / \mathrm{min}$. Failure to respond to certain stimuli, like calling, occurred after the average 2 to 3 minutes. At the beginning of anesthesia AAITM index has varied from 45 to 99 , while the BIS index varied from 91 to 98 . Both parameters have changed with increasing of sedation and end-tidal concentration of sevoflurane. After losing consciousness, BIS ranged 51-79 (average 69), AAITM index 24-85 (average 39). End-tidal concentration of sevoflurane at the moment of loss of consciousness was 2.3 Vol\% (1.3-4.0). Awakening from anesthesia occured in average 5 minutes after the interruption of sevoflurane delivery. On awakening, and in full awareness BIS ranged from 62-80 (average 74 ), while AAITM index was in the range of $14-85$ (average 40). On average the mean value of all BIS values in the awake state was 85 (73-98), in the absence of consciousness 48 (10.83). AAITM index values were on average $71(42-99)$ in the awake state and 21 (4.85) in the absence of consciousness. The study pointed to the fact that neither BIS nor AAITM show a complete correlation with the clinical condition or end-tidal gas concentrations. Cause lies in the factors of time, in pharmacologic and methodological variations. Both indices showed variability with the fact that BIS had significantly less variability than AAITM index ${ }^{38}$.

Another study showed that AAI and BIS are much more relevant in measuring the effects of inhalational anesthesia (sevoflurane, $\mathrm{N}_{2} \mathrm{O}$ ) from composite auditory evoked potentials index $(\mathrm{cAAI})^{39}$.

Ibrahim et al. have examined the ability of BIS to predict depth of sedation between sevoflurane, propofol and midazolam. BIS proved to be a better predictor of sedation with propofol in relation to sedation with sevoflurane and midazolam ${ }^{40}$.

The fact is that in addition to BIS monitoring, which isss commonly used in practice, it is necessary to use all the available information that monitoring of other patient's physiological functions provides, to use own experience and assessment. Bottros et al. used the inverse Turing test to indicate that the anesthetist assessment (who has been trained to recognize and read the clinical and EEG activity) is very close to the BIS monitor. Therefore they have demonstrated the key role of clinicians in monitoring of $\mathrm{AAGA}^{41}$. 
Schneider et al (2014) published a scheme for the integration of BIS information and cardiovascular variables for the production of quantitative multimodal index ${ }^{42,43}$. Depth of anesthesia monitoring can be improved by using standard clinical parameters (heart rate, noninvasive monitoring of blood pressure, oxygen saturation, end tidal concentration of carbon dioxide, respiratory parameters, changes in heart rate and blood pressure), value of the anesthetic concentration (end tidal, concentration of propofol) and patient's demographic data (age, weight, ASA status) besides using EEG monitoring ${ }^{43}$. This combination of factors, EEG and various standard parameters has led to the development of index which is called Anesthesia multimodal index of consciousness (AMIC). AMIC combines variables obtained from EEG, the standard monitoring parameters, each patient's individual data and information on the drugs used. Heart rate and mean arterial pressure are the main parameters which provide information about the cardiovascular system. Some other parameters are: the difference between inspiratory and expiratory pressure ( $\left.\mathrm{Gas} \mathrm{O}_{2} \mathrm{IE}\right)$, end expiratory concentration of carbon dioxide ( $\mathrm{Gas} \mathrm{CO}_{2}$ exp), peak inspiratory pressure (SPIRO Ppeak), end expiratory gas concentrations (MAC exp), age, gender, BMI, etc. The conclusion of this study is that multimodal integration of standard monitoring and EEG parameters can even more accurately reflect the level of anesthesia when compared with only one of these aspects $^{43}$. It is considered that the lack of using this index is in the fact that it is based primarily on the parameters measured and processed by a machine giving a new index parameter. It is unacceptable to use of any kind of "black box" when it comes to patients' lives. It considers that it is better to present monitoring parameters to the clinician, who will then include his/hers experience and make the final decision on the amount of anesthetics and opioids ${ }^{42}$.

\section{Protocols used in practice for avoiding aaga}

Research in the field points to the fact that very few centers have developed protocols for the prevention or treatment of AAGA. It is believed that this is the consequence of AAGA being considered so rare that it is not necessary to develop such a strategy ${ }^{5}$.
In the future depth of anesthesia monitoring would have to integrate EEG data with other external information and anesthesiologist's experience. Appropriate anesthetic technique for awareness prevention relies on careful monitoring of clinical parameters, patient monitoring and regular aquipment checking. Some rules which should be applied in general anesthesia are as follows: patients who did not receive premedication required higher doses of intravenous induction agent, the level of inhalation anesthetics during surgery should be monitored (ETAG), recorded and maintained in order to keep the patient asleep, the level of analgesia should be sufficient to ensure that the patient does not feel pain if consciousness is present, if there is the possibility that awareness is present an amnestic drug (Midazolam) should be used in order to prevent patients memory of pain and events in the operating theatre, it is useful to use earplugs or headphones that transmit music or white noise for the case that the patient semiconscious ${ }^{8}$.

ASA Task Force recommendations on depth of anesthesia monitoring are based on the following ${ }^{44}$ :

- Intraoperative depth of anesthesia monitoring should rely on clinical techniques and conventional patient monitoring. Muscle relaxants can interfere with adequate monitoring of the patient in terms of detecting target or reflex movements.

- Monitoring of the electrical activity of the brain is worth monitoring but it shouldnt be used in assessing the depth of anesthesia in all patients.

- Monitoring of brain function is not used routinely in patients under general anesthesia, nor for routine depth of anesthesia monitoring. Its use is recommended for selected cases of patients who require lower doses of anesthetic, in trauma surgery, caesarean section and total intravenous anesthesia.

If the patient states that he has been aware during surgery, one should take the following actions ${ }^{8}$ : anesthesiologist must visit the patient as soon as possible and examine what the patient remembers, he must review the documentation and check the equipment in order to find possible defects, he must assess patient's condition and explain where the mistake occured, he must note the problem so that future anesthesiologists have that problem in mind when they come in contact with the same patient, he must offer the patient further follow-up as well as psychological help and note that it has been 
offered, he must convince the patient that he can further undergo general anesthesia with minimal risk of future episodes of consciousness. Younger anesthesiologists should inform the competent anesthesiologist about the problem that occurred.

Certainly, the clinician has the obligation to inform the legal department of the hospital, the hospital administration, the patient's general physician, to administer bensodiazepine to patients who are extremely upset.

\section{Conclusion}

The administration of neuromuscular blockers prevents motor response of patients, therefore it takes a lot of experience of the anesthesiologist and adequate monitoring in order to determine the level and state of mind of the patient. The most commonly used monitoring in clinical practice is still BIS monitoring.

\section{References:}

1. Avidan MS, Jacobsohn E, Glick D, et al. BAG-RECALL Research Group. Prevention of intraoperative awareness in a high-risk surgical population. N Engl J Med, 2011; 365:591600.

2. Myles PS, Leslie K, McNeil J, Forbes A, Chan MT. Bispectral index monitoring to prevent awareness during anaesthesia: the B-Aware randomised controlled trial. Lancet 2004; 363:1757-63.

3. Avidan MS, Zhang L, Burnside BA, Finkel KJ, Searleman AC, Selvidge JA, Saager L, Turner MS, Rao S, Bottros M, Hantler C, Jacobsohn E, Evers AS. Anesthesia awareness and the bispectral index. N Engl J Med 2008; 358:1097-108.

4. Pollard RJ, Coyle JP, Gilbert RL, Beck JE. Intraoperative awareness in a regional medical system: a review of 3 years' data. Anesthesiology 2007; 106:269-74.

5. Pandit JJ, Cook TM, Jonker WR, O'Sullivan E. A national survey of anaesthetists (NAP5 Baseline) to estimate an annual incidence of accidental awareness during general anaesthesia in the UK. Anaesthesia 2014; 68:343-53.

6. Griffith D, Jones JB. Awareness and memory in anaesthetized patients. Br J Anaesth 1990; 65:603-7.

7. Kaul HL, Bharti N. Monitoring depth of anaesthesia. Indian J Anaesth 2002; 46(4):323-32.

8. Sinha PK, Koshy T. Monitoring devices for measuring the depth of anaesthesia- an overview. Indian J Anaesth 2007; 51(5):365-81.

9. Mashour GA, Shanks A, Tremper KK, Kheterpal S, Turner CR, Ramachandran SK, Picton P, Schueller C, morris M, Vandervest JC, Lin N, Avidan MS. Prevention of intraoperative awareness with explicit recall in an unselected surgical population. A randomized comparative effectiveness trial. Anesthesiology 2012; 117:717-25.

10. Russel F. The ability of bispectral index to detect intra-operative wakefulness during isoflurane/air anaesthesia, compared with the isolated forearm technique. Anaesthesia 2013; 68:1010-20.

11. Schneider G, Sebel PS. Monitoring depth of anesthesia. Eur J Anaesthesiol Suppl 1997; 15:21-8.

12. Sanders RD, Tononi G, Laureys S. Sleigh JW. Unresponsiveness unconsciousness. Anesthesiology 2011; 116:946-59.

13. Evans JM, Davies WL. Monitoring anaesthesia. Clin Anesth 1984; 2:243-62.

14. Maenpaa M, Penttila J, Laitio T, Kaisti K, Kuusela T, Hinkka S, Scheinin H. The effects of surgical levels of sevoflurane and propofol anaesthesia on heart rate variability. Eur J Anaesthesiol 2007; 24(07):626-33.

15. Russell IF. Fourteen fallacies about the isolated forearm technique, and its place in modern anaesthesia. Anaesthesia. 2013; 68:677-81.

16. Zand F, Hadavi SM, Chohedri A, Sabetian P. Survey on the adequacy of depth of anaesthesia with bispectral index and isolated forearm technique in elective Caesarean section under general anaesthesia with sevoflurane. Br J Anaesth 2014; 112:871-8.

17. Russell IF. Midazolam-alfentanil: an anaesthetic? An investigation using the isolated forearm technique. Br J Anaesth 1993 ; 70:42-6.

18. Sleigh J. The place of the isolated forearm technique in modern anaesthesia: yet to be defined. Anaesthesia 2013; 68:681-3.

19. Pandit JJ, Cook TM. National Institute for Health and Care Excellence guidance on measuring depth of anaesthesia: limitations of EEGbased technology. Br J Anaesth 2013; 110:325-8.

20. Pandit JJ, Cook TM, Jonker WR, O’Sullivan E. A national survey of anaesthetists (NAP5 Baseline) to estimate an annual incidence of accidental awareness during general anaesthesia in the UK. Anaesthesia 2013; 68:343-53.

21. Russell IF. The Narcotrend "depth of anaesthesia“ monitor cannot reliably detect consciousness during general anaesthesia: an investigation using the isolated forearm technique. Br J Anaesth 2006; 96:346-52.

22. Jameson LC, Sloan TB. Using EEG to monitor anesthesia drug effects during surgery. J Clin Monit Comput 2006; 20(6):445-72.

23. Otto KA. EEG power spectrum analysis for monitoring depth of anaesthesia during experimental surgery. Lab Anim 2008; 42(1):45-61.

24. Nunes RR. Entropy: A new method of measuring depth of anesthesia. Comparative study with bispectral index during clinical evaluation in tracheal intubation of patients anesthetized with sevoflurane. Rev Bras Anestesiol 2004; 54(3):289-302.

25. Punjasawadwong Y, Phongchiewboon A, Bunchungmongkol N. Bispectral index for improving anaesthetic delivery and postoperative recovery. Cochrane Database Syst Rev 2007; Issue 4. Art. No:CD003843.

26. National Institute for Health and Care Excellence NICE DiagnosticsGuidance: Depth of anaesthesia monitors. Bispectral index (BIS), E-Entropy and Narcotrend-Compact M. 2012. www.nice.org.uk/dg6 (accessed 29 May 2016).

27. Klopman MA, Sebel PS. Cost-effectiveness of bispectral index monitoring. Curr Opin Anaesthesiol 2011; 24(2):177-81. 
28. Kim JK, Kim DK, Lee MJ. Relationship of bispectral index to minimum alveolar concentration during isoflurane, sevoflurane or desflurane anaesthesia. J Int Med Res 2014; 42(1):130-7.

29. Hodgson PS, Liu SS. Epidural lidocaine decreases sevoflurane requirement for adequeste depth of anesthesia as measured by the Bispectral Index Monitor. Anesthesiol 2001; 94:799-803.

30. Panchal NN, Swadia VN, Patel MG. Correlation of BIS index with sevoflurane concentration in paediatric anaesthesia. Nat J Med Res 2014; 4(2):156-60.

31. Renna M, Handy J, Shah A. Low baseline Bispectral Index of the electroencephalogram in patients with dementia. Anesth Analg 2003; 96:1380-5.

32. Galante D, Fortarezza D, Caggiano M, de Francisci G, Pedrotti D, Caruselli M. Correlation of bispectral index (BIS) monitoring and end-tidal sevoflurane concentration in a patient with lobar holoprosencephaly. Rev Bras Anestesiol 2015; 65(5):379-83.

34. Kreuer S, BiedlerA, Larsen R, Schoth S, Altmann S, Wilhelm W. TheNarcotrend ${ }^{\mathrm{m}}$ - a new EEG monitor designed to measure the depth of anaesthesia. A comparison with bispectral index monitoring during propofolremifentanil-anaesthesia. Anaesthesist 2001; 50:921-5.

35. Schneider G, Kochs EF, Horn B, Kreuzer M, Ningler M. Narcotrend ${ }^{\circledR}$ does not adequately detect the transition between awareness and unconsciousness in surgical patients. Anesthesiol 2004; 101:1105-11.

36. Russell IF. The Narcotrend 'depth of anaesthesia' monitor cannot reliably detect consciousness during general anaesthesia: and investigation using the isolated forearm technique. Br J Anaesth 2006; 96(3):346-52.

37. Chen X, Tang J, White PF, Wender RH, Ma H, Sloninsky A, Kariger R. Acomparison of patient state index and bispectral index values during the perioperative period. Anesth Analg 2002; 95:1669-74.

38. Wong CA, Fragen RJ, Fitzgerald P, McCarthy RJ. A comparison of the SNAP II and BIS XP indices during sevoflurane and nitrous oxide anaesthesia at 1 and 1.5 MAC and at awakening. Br J Anaesth 2006; 97:181-6.

39. Anderson RE, Barr G, Assareh H, Jakobsson J. The $\mathrm{AAI}_{\mathrm{TM}}$ index, the BIS index and end-tidal concentration during wash in and wash out of sevoflurane. Anaesthesia 2003; 58:531-5.

40. Nishiyama T. Composite-, plain-auditory evoked potentials index and bispectral index to measure the effects of sevoflurane. J Clin Monit Comp 2013; 27(3):335-9.

41. Ibrahim AE, Taraday JK, Kharasch ED. Bispectral index monitoring during sedation with Sevoflurane, Midazolam and Propofol. Anesthesiol 2001; 95:1151-9.

42. Bottros MM, Palanca BJA, Mashour GA, Patel A, Butler C, Taylor A, Lin N, Avidan MS. Estimation od the bispectral index by anesthesiologists: An inverse Turing test. Anesthesiol 2011; 114:1093-101.

43. Sleigh J. No monitor is an island: depth of anaesthesia involves the whole patient. Anesthesiol 2014; 120:799-800.

44. Schneider G, Jordan D, Schwarz G, Bischoff P, Kalkman CJ, Kuppe H, Rundshagen I, Omerovic A, Kreuzer M, Stockmanns G, Kochs EF; European Multicenter EEGAEP Anesthesia Monitoring Study Group and Research Group Knowledge-based Signal Processing. Monitoring depth of anesthesia utilizing a combination of electroencephalographic and standard measures. Anesthesiol 2014; 120:819-28.

45. American Society of Anesthesiologists Task Force on Intraoperative Awareness. Practice advisory for intraoperative awareness and brain functionmonitoring, A Report by the American Society of Anesthesiologists Task Force on Intraoperative Awareness. Anesthesiology 2006; 104:847-64. 